\title{
The optimization of calibration of the computer model describing the flows in the water distribution network; Study of the example
}

\author{
Maria Orłowska-Szostak ${ }^{1, *}$ \\ ${ }^{1}$ Faculty of Civil and Environmental Engineering, Gdansk University of Technology, \\ ul. G. Narutowicza 11/12, 80-233 Gdansk, Poland
}

\begin{abstract}
The paper describes the issues of optimization of calculations performed during the calibration of a computer model describing the flow in the water distribution network. Optimization concerns the efficiency of these calculations which means not only the volume and speed of calculations, but above all, the optimal use of the measurements made during calibration to obtain the best, i.e. the most real value of the searched parameters/characteristics of the system components. The paper includes a short introduction which orders the range of methods of calibration and the area of calculation methods used in this class of issues, and then the description of an example where in the calibration process was used a computational tool called EPANET Calibrator developed by scientists - engineers of cooperating Brazilian universities. This tool is based on genetic algorithm.
\end{abstract}

\section{Introduction}

Calibration of the model describing the phenomenon of flows occurring in the system of transport and distribution of water intends to define the characteristics of system components (parameters appearing in their analytic descriptions), and so to ensure that the model shall describe this phenomenon with required accuracy. The aim of calibration is not to measure the whole system, because in this case we would know everything we want about the system and computer modeling could lose its sense because it would often be enough to monitor the results of measurements. During the calibration process one carries out measurements only at the selected/possible optimal points/ nodes of the system (see, e.g.: [1]), and then the results of these measurements are used to determine the possible optimal characteristics/parameters (the methods of performance at these step of creation of a computer model are described later in the paper) and thanks to that it is created a calibrated model, which is a tool for simulating of all possible real behaviors of the system. facts:

The calibration process is characterized by indeterminacy resulting from the following

- mentioned, rarely distributed measurement points,

\footnotetext{
Corresponding author: maria.o@pg.gda.pl
} 
- the fact that they are not known either searched characteristics of the system components (especially the roughness of pipes) and flows in the network (or water partitions) occurring during performing these measurements.

There are used different calibration methods, and the aim of each of them is the removing (reducing the value) the said indeterminacy of the task. Each method requires, for example, performing a variety of measurements used in the calibration. Frequently used method of reducing the number of unknown roughness and partitions [2] is a grouping of pipes of virtually identical roughness coefficients and grouping of junction nodes with virtually identical timing patterns of water partitions. The criteria for the creation of such groups are not always convincing, although this method is widely used.

The efficiency of the calibration also depends on the method of performance (intuitive ones, methods/algorithms implemented numerically) for the optimal use of the above mentioned measurements in the calibration of the model, i.e. as mentioned above, in the determination of characteristics (parameters appearing in the analytical descriptions of these characteristics) of the system components.

Among the above-mentioned calibration methods, the most popular are the following ones:

(1) method, which can be called "direct"

This method uses measurements of pressure and flow at selected points of the system. Generally, the pressures are measured for calibration purposes at pipe junction selected nodes (not at all nodes) of the water distribution network. In small and medium-sized systems the junction nodes for measuring are selected intuitively (as described later in this paper, in the description of the calibration carried out for the example water distribution system). For the larger systems, it is reasonable to optimize the number and location of junction nodes at which we perform the measurements (see e.g. the theoretical basis of sampling design [1]). In each case, the flows are usually measured at all objects, such as pumping stations of water intakes, the zonal pumping stations, storage tanks and finally in selected pipes. The weakness of the described calibration method is an unknown water partitions patterns occurring at the time of the measurements. This is the reason of the strong indeterminacy of the whole task of calibration. We try to "arrange" the partitions of water as reliably as possible between the junction nodes, so that their sum was compatible in the each zone with the measured supplies in these zones, and at the same time to use all available data and information on the consumption of water by individual customers. The determination of simultaneous partitions of water for all customers during performing the aforementioned measures of pressure and flows, without taking (practically impossible to perform) continuous measurements at those customers, was not possible. Therefore, the accuracy of the calibration was limited and difficult to estimate. However, during long use of so calibrated model it was possible further verification of its parameters, which caused that the designated roughness of the pipes was treated with more and more confidence. From the other hand, applying genetic algorithms for the measuring data made with the 'direct' method is very much prospering what was shown in the article.

(2) the fire hydrant flow test

This method better cope with the described indeterminacy of calibration. It is tried to bring to the situation in which we would be practically very close to determination of water consumption in the supplied area, with simultaneous values of pressures and flows measured as in the previous method. This method is based on taking identical measurements of pressures and flows and the estimation of consumption of water in the supplied area, as in the previous method, except that:

- it is considered and measured the specific night situation at the hour of minimum consumption, close as possible to zero consumption, 
- when making the above-mentioned measurements, one or more, usually several fire hydrants are fully open, and at the same time their discharges and pressures are measured.

The discharges of opened hydrants should be significant/dominant water distributions in the system during measurements needed for calibration; other, "night" distributions are low. In this way we are approaching a situation where we know the consumption occurring during measurements, and therefore we can substantially reduce the indeterminacy of calibration which is characteristic in the previous method.

(3) the tracer test

Tracer test methods of the calibration of models are the most developed in hydrology, in modeling of groundwater flow. The use of tracers method in the calibration of models describing flows in the water distribution systems is based, in addition to measurements performed as in "direct" method, on introducing a specific quantity of tracer to the network, especially at its points of supply. By measuring the concentration of this tracer in many points on the network, using the proper equipment, and assuming generally correct assumption that the distribution of tracer in the network can be described with the piston model and the amount of tracer flowing in and flowing out in the junction node are equal, it is measured the speed of flow in the water network, considerably reducing the indeterminacy which is characteristic for the "direct" method (see above). Calibration accuracy depends here on the density and location of the measurement points, whereas in the "direct" method characterized by a lack of real, current data describing the water consumption during calibration, described indeterminacy of the task occurs.

It should be emphasized that very good results of the calibration of model considering the roughness of pipes is ensured by fire hydrant flow test, because during the calibration process using this method it is not necessary to determine the accurate values of consumption of water or to determine the timing patterns of such consumption. Therefore, sometimes presented approach to the calibration of the model [2] involves the use of the fire hydrant flow test (where it is simulated a single technical situation in the system using SPA - Single Period Analysis), and additionally, if we are interested in consumptions in the supplied areas (their values along with timing pattern), then at already calibrated roughnesses it is performed a calibration of the said consumption patterns using e.g. the tracer method or using the results of continuous monitoring of pressures and flows. It is applied here the space-time simulation EPA - Extended Period Analysis.

Let's consider these methods applied for the optimal use of the above-mentioned measurements in the calibration process. The task involve the solving of nonlinear systems of equations describing the behavior of the system (implicit constraints in the model) and searching of the roughness vector of the pipes (group of pipes) and sometimes the consumption vector i.e. decision vectors ensuring the global minimum of the objective function. That objective function is constructed so that by using the method of the least squares of differences between the measured and simulated values it could be possible to determine the actual values of searched roughness and, if necessary, consumption [3]. Solving of the mentioned system of equations of conservation is a known issue. They are applied known, classical numerical methods (Newton-Raphson method for nodal pressures corrections, gradient method) and heuristic methods, which include genetic algorithms [4].

Taking into account the methods of searching for the vector minimizing the value of the said objective function, it must be emphasized that in comparison to the classical numerical methods, computer heuristic methods protect against the determination of a local minimum treated as a global minimum. Therefore (but not only therefore), they are more and more often used by engineers. Regarding the issues and their resolving, which are the essence of the calibration of the model of water distribution network the best are genetic algorithms $[2,5,6]$. 
This paper will describe a computer model of flows occurring in the sample water distribution network. The model was created using the EPANET program. It was described the calibration of the model using the above-mentioned direct method, based on the measured pressures and flows in designated points of the water distribution system.

\section{The analyzed exemplary water distribution system}

The water supply system consists of the water intake and treatment station working in a two-stage pumping system. The water intake is located in the central part of the city and consists of eight deep-water wells. Water is drawn from the decks of quaternary aquifers [7]. The total yield of the wells is $440 \mathrm{~m}^{3} / \mathrm{h}$ with depression from 7.5 to $16 \mathrm{~m}$. Water is taken alternately from individual groups of wells to ensure the required time-varying capacity of the intake. Water from drilled wells is pumped by the first stage submersible pumps to the treatment plant. Once treated, the filtered water flows into reservoirs of clean water. Two field tanks, with a volume of $2,100 \mathrm{~m}^{3}$ each, serve as storage tanks in the water supply system [8]. The dispatcher maintains a constant water level in the tank at a height of $3.7 \mathrm{~m}$, measured from the bottom of the tank. The second stage pumps draw water from the tanks and pump it to the network. These pumps are controlled by VSD to provide the desired pressure at the outlet of the pump station: 4.5 bar during the day and 4.0 bar overnight. These values were appointed by the dispatcher of the system basing on his exploitation experience, however they fulfill all of the major constraints given in professional literature, see e.g. [9].

The water supply network is a loop-type with few branched network elements. The outline of the analyzed water supply system, executed in the EPANET program, was shown in Fig. 1. The structure of the network, including the materials that the pipes are made of, is characteristic of typical urban networks in Poland [10]. Cast iron pipes account for more than half of all urban network pipes. A significant part of the network was also made of PVC [8]. The age structure of the network has been carefully worked out by the authors because it is an important factor in determining the initial roughness of the pipes during model calibration. Cast iron pipes are the oldest types here. Information was also gathered on ongoing flushes and other regenerative pipe processes as they have a significant impact on the pipes roughness [11].

\section{Basic data for the model of water distribution system}

Carefully gathered data and standard software based on EPANET were used for construction of the computer model. Specifically, data describing the consumption of water and the network topology were collected, as well as any necessary parameters characterizing it.

When determining reliable water consumption, the results of continuous measurements of the second-stage pump station delivery, pumping water directly to the network were set as the starting point. Base consumptions and time patterns of inequality of hourly water consumption for individual connections were determined with taking into consideration some specific information about water consumption of individual objects or more often the sizes of the objects, their character, and sometimes the diameter of the connections themselves. 


\section{Applied method of calibration of model, applying a genetic algorithm with respect to the analyzed water distribution system}

Taking into account the assortment of measurements, the applied method of the calibration of model can be classified as the direct method, described in chapter 1, i.e. the method which uses the measurements of pressure and flows at selected points of the water distribution system.

The drawing up of the results of above-mentioned measurements, i.e. estimation of roughness of pipes forming a water distribution network as well as emitter coefficients was carried out using a genetic algorithm. It was applied EPANET Calibrator software, developed by Resan Labs from Brazil [3,6,12].

The authors of the EPANET Calibrator software give the form of the objective function in optimization task of calibration of model of the system of transport and distribution of water. Therefore, the criterion of the optimization task is to minimize the said objective function [12]

$$
\operatorname{MinFO}=\sum_{i=1}^{N}\left[\frac{\left.\frac{\Delta P_{i}}{N}\right]^{N} P_{i}^{\prime}}{N}\right]^{2}+\sum_{j=1}^{M}\left[\frac{\Delta Q_{j}}{\frac{\sum_{j=1}^{M} Q_{j}^{\prime}}{M}}\right]^{2}
$$

where: $P^{\prime} i$ - set of pressure values, $Q^{\prime} i$ - set of flow values, $\triangle P i$ - difference of pressure between simulated and observed data, $\Delta Q i$ - difference of flow between simulated and observed data, $N$ - number of monitored nodes, $M$ - number of monitored pipes.

The coordinates of the decision vector can be roughnesses of pipes and emitter coefficients, which in the objective function occur in the implicit form. In turn, implicit restrictions in this optimization task are conservation equations describing the flows in the system. Due to formal reasons, mentioned coordinates of the decision vector are under restrictions with explicit character. The optimal solution is the decision vector meeting the above criterion.

The consumption of water in time and space of the supplied area and also the initial roughnesses of pipes have been determined as precisely as possible due to their high importance for the convergence and the stability of the algorithm. Localization of measuring points was made following the selection of sites with the highest pressure fluctuations (primarily terminal nodes of the network branches) and in the areas with the largest water consumptions (around the "centre of gravity of the system").

Schematic visualization of the water supply system modeled by us was shown on the Fig. 1. In this picture groups of pipes were marked; how we recalled in chapter 1, we are creating the groups of pipes of similar roughness in order to reduce the number of unknowns / searched roughnesses. 


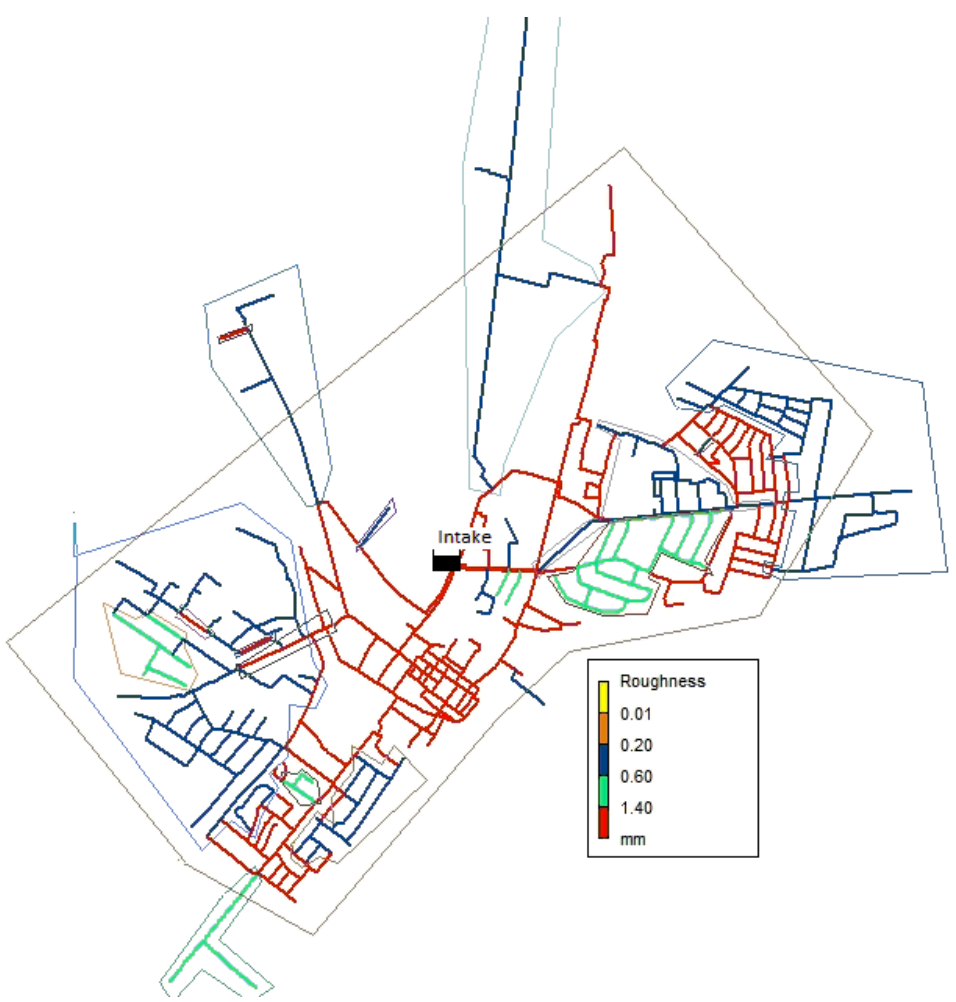

Fig. 1. Scheme of the modeled water supply system with groups of pipes marked by their roughnesses.

Essential findings and remarks about calibration are given in the last chapter devoted to conclusions. Only brief calibration results are provided in this chapter, which ensure that the model adequately describes the operation of the water supply system.

Fig. 2 shows the comparisons of observed $24 \mathrm{~h}$ pressures with simulated ones before and after calibration, at two exemplary nodes.
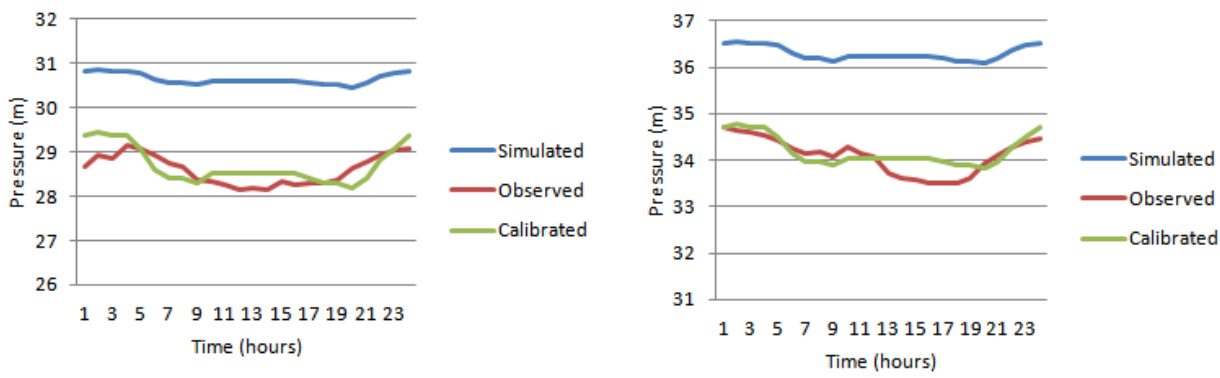

Fig. 2. Comparison of observed pressures with simulated ones before and after calibration, at exemplary nodes; Initial population: 200 , Generations: 400, Mutation probability: 0,01, Crossover probability: 0,9 .

As can be seen, as a result of the performed calibration, i.e. estimation of the pipes roughnesses and the determination of the emitters coefficients, the accuracy of the system operation simulation is very good, what was not possible to be stated with the reference to not-calibrated model, although starting values of the roughnesses of pipes and the partitions 
of water were assumed with the great care. Differences between pressures simulated with the help of calibrated model and pressures that were measured do not exceed permissible values given at the work [13], and they even are definitely smaller.

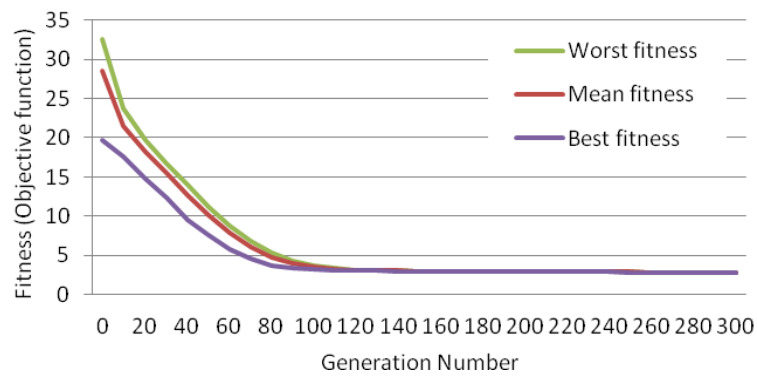

Average Distance Between Idividuals

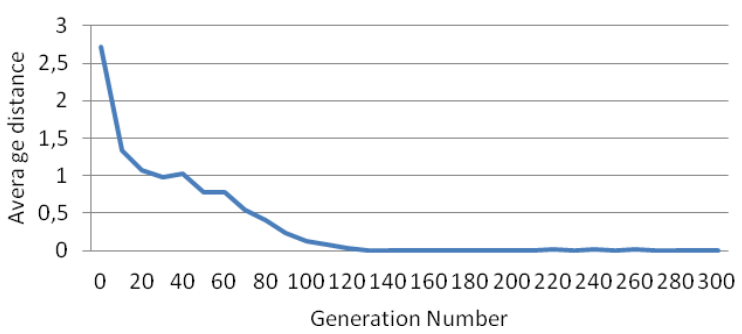

Fig. 3. Characteristics of the convergence of the applied genetic algorithm.

As can be seen, calculations conducted with the EPANET Calibrator software were not only stable, but also were characterized by a very good convergence. In contrast with often applied "manual" roughnesses adjustments while making the calibration, these calculations are proceeding automatically and quickly, not requiring the interference of the engineer drawing the computer model up. It also helps to avoid the tendency of setting the local minimum of the objective function.

\section{Conclusions}

1. The author carried out a calibration of the model using the "direct" method, i.e. using measurements of pressure and flow in selected locations of the system. Thanks to applying presently standard methods of reducing the number of unknown roughnesses, as well as thanks to advantages of the genetic algorithm, the direct method of calibration turned out to be completely sufficient.

2. It is important to choose the proper localization of the measuring points. The method of locating these points described in the article was sufficient. More sophisticated methods of sampling design are desirable only for large and complex water supply systems.

3. In the process of the calibration we are measuring both pressures and flows and at set partitions of water the result of the calibration exploiting the EPANET Calibrator software is a vector of roughnesses of pipes and emitters coefficients.

4. Significant elements affecting the quality (convergence) of the calibration of the model are: carefully determined water consumptions of the historical period used for the calibration as well as carefully determined demand patterns. It is also the condition for estimating possibly accurate leakages. 
5. The graph presented in the Fig. 3 is demonstrating a very good, better than assumed while starting the calibration, convergence of the optimization process, which is a contribution of both the genetic algorithm implemented in the EPANET Calibrator software and carefully prepared starting data recalled in the previous chapter.

6. Applying described method of calibration and software based on EPANET, i.e. EPANET Calibrator utilizing the genetic algorithm, the computer model providing very good accuracy of the system operation simulation was obtained. Differences between pressures simulated with the help of calibrated model and pressures that were measured do not exceed permissible values given at the work [13], and they even are definitely smaller.

\section{References}

1. C. A. Bush, and J. G. Uber, J Water Res Pl-Asce, 1249,6, 334-344 (1998) (http://dx.doi.org/10.1061/(ASCE)0733-9496(1998)124:6(334))

2. S. Lingireddy and L. E. Ormsbee, Civ Eng Environ Syst , 19, 1, 13-39 (2002) (http://dx.doi.org/10.1080/10286600212161)

3. N. Abe, P. B. Cheung, EPANET Calibrator. User Guide. (2008)

4. E. Keedwell, Soon-Thiam Khu, Eng Appl Artif Intel, 18, 461-472 (2005) (http://dx.doi.org/10.1016/j.engappai.2004.10.001)

5. Di Nardo, M. Di Natale, C. Gisonni, M. Iervolino, J Water Supply Res T 64,1, 36-46 (2015) (http://dx.doi.org/10.2166/aqua.2014.004)

6. W. Stanisławski, M. Zmarzły, Pomiary Automatyka Robotyka,12, 61-64 (2010)

7. A.Wróbel, Comprehensive analysis of the operation of water supply network, for example for the town of Dziatdowo, using a computer model of the network created in Epanet 2.0, Master's thesis done under the direction of M. Szostak-Orłowska, Department of Sanitary Engineering, Faculty of Civil and Environmental Engineering, Gdańsk University of Technology, Poland (2015), in Polish

8. D. Sarnowski, Gospodarka wodno-kanalizacyjna w Działdowie, Działdowo, Poland (2012), in Polis

9. V. Ghorbanian, B. Karney and Y. Guo, J Water Res Pl-Asce,142,8 (2016) (http://dx.doi.org/10.1061/(ASCE)WR.1943-5452.0000665)

10. M. Kwietniewski, M. Tłoczek, L. Wysocki et al., Rules for selection of materials and design solutions for the construction of water pipes,(Publishing House Izba Gospodarcza Wodociągi Polskie, Bydgoszcz, Poland, 2011), in Polish

11. Cz. Grabarczyk, Liquid flow in pipes: calculation methods, (Publishing House Envirotech Sp. z o.o., 1997), in Polish

12. Narumi Abe, P. B. Cheung, Epanet Calibrator - An integrated computational tool to calibrate hydraulic models, (Integrating Water Systems. Boxall \& Maksimovic (eds) Taylor \& Francis Group, London, ISBN 978-0-415-54851-9, 2010)

13. T. M. Walski, D. V. Chase, D. A. Savic et al., Advanced water distribution modeling and management, (Haestad Press, Waterbury, CT USA, 2003)

14. L.A. Rossman, EPANET 2 Users Manual, (EPA, United States Environmental Protection Agency, Cincinnati, 2000) 